\title{
Monitoring micrometer-scale collagen organization in rat-tail tendon upon mechanical strain using second harmonic microscopy
}

\author{
Y. Goulam Houssen ${ }^{1}$, I. Gusachenko ${ }^{1}$, M.-C. Schanne-Klein ${ }^{1}$ and J.-M. Allain ${ }^{2}$ \\ ${ }^{1}$ Ecole Polytechnique, Laboratory for Optics and Biosciences, 91128 Palaiseau, \\ France; CNRS, 91128 Palaiseau, France; INSERM U696, 91128 Palaiseau, France. \\ ${ }^{2}$ Ecole Polytechnique, Solid Mechanics Laboratory, 91128 Palaiseau, France; \\ CNRS, 91128 Palaiseau, France. \\ Corresponding author: Jean-Marc Allain \\ Ecole Polytechnique, Solid Mechanics Laboratory, 91128 Palaiseau, France; \\ CNRS, 91128 Palaiseau, France. \\ Tel: +33-1-69335812 \\ Fax: +33-1-69335706 \\ E-mail : allain@lms.polytechnique.fr
}

\begin{abstract}
We continuously monitored the microstructure of a rat-tail tendon during stretch/relaxation cycles. To that purpose, we implemented a new biomedical device that combined SHG imaging and mechanical testing modalities. This multi-scale experimental device enabled simultaneous visualization of the collagen crimp morphology at the micrometer scale and measurement of macroscopic strain-stress response. We gradually increased the ultimate strain of the cycles and showed that preconditioning mostly occurs in the first stretching. This is accompanied by an increase of the crimp period in the SHG image. Our results indicate that preconditioning is due to a sliding of microstructures at the scale of a few fibrils and smaller, that changes the resting length of the fascicle. This sliding can reverse on long time scales. These results provide a proof of concept that continuous SHG imaging performed simultaneously with mechanical assay allows analysis of the relationship between macroscopic response and microscopic structure of tissues.
\end{abstract}

Keywords: Second Harmonic Generation, collagen, preconditioning, mechanical properties, tendon.

\section{Introduction}

The relationship between microscopic macromolecular organization and macroscopic biomechanical properties is a general concern in collagenous tissues. Tendon is a model system in that respect: it is almost unidirectional and does not require biaxial loading in biomechanical studies. It is a highly structured tissue designed for force transmission from muscle to skeleton, mainly composed of type I collagen that forms fibrils of around $200 \mathrm{~nm}$ diameter. These fibrils assemble into fibers that further form fascicles with a crimped pattern (Kastelic et al. 1978). This hierarchical organization is responsible for the biomechanical properties of the tissue.

The macroscopic mechanical properties of tendon are fairly well known (Abrahams, 1967; Ker, 2007). Quasi-static stretching of rat-tail tendon gives a non-linear response, with a toe region in the first few percent of strain, followed by a linear region. However, the relationship between these properties and the structure at lower scales (such as fibers or fibrils) remains a subject of strong interest (Puxkandl et al., 2002; Fratzl, 2003; Screen et al., 2004; Gupta et al., 2010). The initial toe region is attributed to a straightening of the initially crimped collagen fibrils (Rigby et al., 1959; Viidik \& Ekholm, 1968; Hansen et al., 2002; Franchi et al., 2007). The linear region is considered as the response when the collagen fibrils are aligned in the direction of traction, so it is associated with an extension of collagen fibers combined with a sliding of the fibrils 1 
in their proteoglycan matrix (Fratzl, 2003). Tendons subjected to cycles of stretch/relaxation exhibit a hysteretic stress-strain relationship (Fung, et al., 2009). This is attributed to a rearrangement of the proteoglycan matrix around the collagen fibrils (Puxkandl et al., 2002; Redaelli et al., 2003).

Further insight into these microscopic processes requires continuous monitoring of tendon microstructure during mechanical assays. In that respect, few studies using semi-continuous optical imaging of tendon stretching have been reported. Confocal microscopy has been used to track labeled cell nucleus in tendons (Screen et al., 2004). Optical Coherence Tomography (OCT) has been used to monitor the straightening of tendon crimps with applied tensile strain (Hansen et al., 2002). The later technique provides images of unlabelled collagen fibrils, but with limited contrast because of its low specificity. In that context, Second Harmonic Generation (SHG) microscopy is a valuable technique to investigate the microstructure of collagenous tissues. SHG is a multiphoton mode of contrast at exactly half the excitation wavelength that is highly specific for dense noncentrosymmetric media, such as fibrillar collagen (Freund et al., 1986; Campagnola et al., 2002; Stoller et al., 2002; Zoumi et al., 2002; Cox et al., 2003; Zipfel et al., 2003; Strupler, et al., 2007; Deniset-Besseau et al., 2009). As a coherent nonlinear optical process, it is an effective structural probe of the micrometer-scale collagen macromolecular organization (Pena et al., 2005; LaComb et al., 2008; Rivard et al., 2011). Moreover, it is characterized by intrinsic three-dimensional (3D) resolution like two-photon excited fluorescence (2PEF). SHG microscopy hence enables 3D visualization of intact tendons by recording stacks of optically sectioned images with typically 1-3 $\mu \mathrm{m}$ axial resolution. It has been shown to provide high-contrast images of the characteristic crimped pattern of this tissue (Stoller et al., 2002; Stoller et al., 2003; Williams et al., 2005; Erikson et al., 2007; Legare et al., 2007; Gusachenko et al., 2010). SHG imaging has recently been used to visualize damage in tendons after fatigue tests, and proved efficient at providing 3D maps of fiber orientations (Fung et al., 2010).

This paper reports the development of multi-scale experiments to monitor simultaneously the microstructural organization of tendon and its macroscopic mechanical response. To that end, we perform mechanical measurements while imaging optical sections of a whole tendon fascicle using SHG microscopy and analyzed the stress-strain relationship versus the crimp morphology. To the best of our knowledge, continuous monitoring of tendon microstructure during multiple loading cycles has not been reported yet. We observe that cyclic stretch/relaxation at increasing strains leads to a shift of the toe region accompanied by an increase of the crimp spatial period. Our data confirm that the toe region is due to the straightening of the crimps, and that the linear region is due to the fibrils sliding.

The paper is organized as follows. The next section is devoted to a detailed description of the experimental methods. Then we present our mechanical measurements and SHG images for increasing cyclic stretching. We finally discuss the mechanical data through changes of the microstructure morphology.

\section{Materials and Methods}

\section{Rat-tail tendon preparation and clamping to the testing device}

The tendons were extracted from Sprague Dawley rat tails (female, 250-300 g, $n=3$ ). The tails were kept frozen until dissection. Incisions were made at the tapered end of the tails using pliers to pull out the tendon fascicles gently. The tendons were rinsed in phosphate buffered saline (PBS) and centrifuged at $4700 \mathrm{rpm}$ (3 $\times 10$ mins) to remove all other tissue components. The tendon fascicles were stored in $\mathrm{PBS}$ at $4^{\circ} \mathrm{C}$ and used within a few days for the experiments.

Tendon fascicles were attached to the testing device as depicted in Figure 1. They were glued with cyanoacrylate onto a metallic plate $\left(20 \times 20 \times 1 \mathrm{~mm}^{3}\right)$ and coiled on rods inserted in the plate, in an almost symmetric manner to limit any boundary effect due to gluing (see Fig. 1C). The fascicles were suspended vertically and allowed to rotate freely before gluing to a second plate, to minimize initial torsion. We always gently manipulated the tendon fascicles to avoid uncontrolled stretching and maintained them in PBS to prevent drying (except for the ends). The plates were then firmly clamped to small screw-tightened grips within the testing device. The distance between the grips was adjusted to maintain the tendon fascicles almost horizontal, with 1-2 mm of deflection at most. This initial deflection is due to the fascicle weight and 
cannot be avoided without stretching the fascicle. During all experiments, the tendon fascicles were immersed in PBS using a glass cover-slip just under the sample (see Fig. 1A and B). In some experiments, the tendon fascicle was labeled with fluorescent latex beads (1 $\mu \mathrm{m}$ diameter, L1030, Sigma-Aldrich) to monitor local strains.

\section{Multiphoton imaging}

The tendon microstructure was observed by putting the testing device in place of the stage in a custom-built laser scanning microscope (see Fig 1A and B) (Strupler et al., 2007). Tendons were illuminated in an upright geometry using circularly-polarized excitation at $860 \mathrm{~nm}$ from a femtosecond titanium-sapphire laser (Spectra-Physics). SHG and 2PEF signals were recorded in 2 photon-counting epi-detection channels using appropriate dichroic mirrors and spectral filters (Strupler et al., 2007). We used a 20×0.95 NA water objective with typically $0.40 \mu \mathrm{m}$ (lateral) $\times 1.6 \mu \mathrm{m}$ (axial) resolution near the sample surface. SHG images were recorded using $200 \mathrm{kHz}$ pixel rate and $0.8 \mu \mathrm{m}$ pixel size, with typically $50 \mathrm{~mW}$ excitation power. We verified that we always imaged the same region of the tendon fascicle by using characteristic patterns in the SHG images as references. When necessary, we slightly shifted the microscope stage to compensate for small lateral displacements during loading cycles. Adjustment of the objective focus was usually required because of slight axial displacement of the tendon fascicle. In some experiments, z-stacks of combined SHG and 2PEF images were acquired along the full thickness of the tendon fascicle (every $2 \mu \mathrm{m}$ ). No photodamage of the tendon was observed under these experimental conditions.

SHG images were analyzed using ImageJ software (W. Rasband, NIH) to estimate the crimp period (distance measurements along the tendon axis).

\section{Mechanical testing}

Mechanical tests were performed at room temperature using a uniaxial RAITH® testing device. The force was measured using a 10N load cell (XFL225D10, FGP Sensors) with 1\% accuracy. Stress was obtained as the force divided by the initial cross-sectional area, assuming a cylindrical shape of the tendon fascicle. Typical diameter was $400 \mu \mathrm{m}$, as obtained from SHG image-stacks recorded before stretching. Strain was obtained as the ratio of the grips displacement divided by the initial length of the tendon fascicle (typically $26 \mathrm{~mm}$ ). Displacement and force values were measured every second. The fascicles were stretched at $10 \mu \mathrm{m} / \mathrm{s}$ constant strain rate (around $3 \times 10^{-2} \% \mathrm{~s}^{-1}$ ).

To investigate possible slipping, we compared the grips displacement to the local displacement on the tendons through the tracking of ink labels, separated by $10 \mathrm{~mm}$ and imaged under a binocular microscope. No significant difference was observed. However, we noticed that significant torsion could occur if the tendon fascicle torsion was not carefully minimized before gluing.

Typical loading path was composed by successive series of two stretch/relaxation cycles at the same ultimate strain (see Fig 2A). The ultimate strains of the series were progressively increased up to breaking. The traction device was immobilized during image acquisition.

\section{Results}

\section{Stress-strain relationship}

Typical strain-stress measurements are displayed in figure 2. Fig. 2A shows the loading path made up of successive series at increasing ultimate strains (2, 4, 5, 8, and 12\%). Each point corresponds to a typically 40s pause for SHG imaging. Fig. 2B shows the measured stresses for the whole loading path. We observe similar stress-strain curves to those previously reported (Rigby et al., 1959), with an initial toe-region followed by a linear part at larger strains - which can be clearly defined in our case as strains higher than $6 \%$. The curves shift to the right as we gradually increase the ultimate strain in the series 
example, the curves for $5 \%$ ultimate strain - in blue - seem to be a translation to higher strains of the curves for $4 \%$ ultimate strain - in green. The only exception to this observation is the last curve (magenta). We attribute the backward shift at $12 \%$ to a longer pause (about 5 minutes) at $0 \%$ strain before stretching to 12 $\%$.

The observed stresses are quite reproducible in our experiments but they are significantly lower than previously reported ones (Hansen et al., 2002; Sionkowska and Wess, 2004; Screen et al., 2005). The slope of the linear part gives an equivalent Young modulus of $75 \mathrm{MPa}$ (considering the last stretching up to $12 \%$ strain). This quantitative discrepancy may come from uncontrolled strain application during fascicle preparation. We think however that it is most likely due to the storage in PBS solution, which was reported to lead to fascicle swelling and subsequent artificial increase of the fascicle cross-section for stress calculation (Legerlotz et al., 2010). Moreover, we corroborated that fresh tendon fascicles give similar results to previously frozen ones. Our setup also enabled us to monitor the motion of the tendon surface by imaging fluorescent beads. We verified in this way that there was no excessive torsion and that the macroscopic strain corresponded to the local strain, at least for the first stretching.

Figures $2 \mathrm{~B}$ and $\mathrm{C}$ focus on the stress-strain relationship for the series with ultimate strains of $5 \%$ and $8 \%$ respectively. We also include, for clarity, the last relaxation of the previous series (in black) and the first stretch of the next series (in gray). The set of curves illustrates preconditioning: the curves shift slowly to the right, reaching lower stresses for the same strain for the second cycle. The modification of the stress-strain relationship is strongest during the first stretch of a series. The stresses in the first relaxation and in the second cycle at this ultimate strain are very close, and cannot be easily distinguished given our sensor resolution. Thus we performed only 2 cycles per series at a given ultimate strain.

To quantify more precisely the preconditioning, we measured the maximum stress reached in each cycle (Fig 2E) and the minimal strain at which we could measure a significant stress (Fig 2F). These two quantities could be defined in all the cycles, whereas we could not determine a linear part for cycles with small ultimate strain. As there are 2 cycles per series, there are 2 maximum stresses per ultimate strain in figure 2E. The maximal stress of the second cycle of a series is always smaller than that of the first cycle, illustrating preconditioning. As expected, we see that the ultimate stress increases with the ultimate strain, although this increase is not homogeneous - for example, the ultimate stresses for $4 \%$ and $5 \%$ ultimate strains are similar.

Figure 2F displays the lowest strains at which the stress is significantly different from the noise versus the ultimate strain. This quantity characterizes the onset of the toe-region of the stress-strain curve. As strain is measured with respect to the initial length of the fascicle, it can be considered as the change in the resting length of the tendon fascicle: the larger the lowest strain, the longer the fascicle. Since a series includes two stretches and two relaxations, there are four points for each ultimate strain, although in most cases the last three are indistinguishable. We call this value the limit lowest strain for a given series. It is reached after the first stretch for each series and increases linearly with the ultimate strain of the series (dotted line in Fig 2F). Linear fitting gives a slope of 0.58 and a lowest strain of $0.44 \%$.

Fig $2 \mathrm{~F}$ appears as a stair-shaped curve. The lowest strain indeed increases abruptly between the first stretch and the first relaxation in a series. Then, when the ultimate strain is increased for a new series, the first stretch has not been preconditioned, and the lowest strain does not change. Consequently, the points are almost indistinguishable and the curve in Fig 2F moves horizontally. The last point at $12 \%$ ultimate strain is lower since we allowed the tendon fascicle to relax for 5 minutes.

\section{Crimp evolution}

In order to connect the macroscopic biomechanical behavior of tendon to its microscopic collagen organization, we paused and recorded SHG images at every point on the loading path. Typical images are displayed in Fig. 3A-E and correspond to positions emphasized by orange circles in the loading path depicted in Fig. 3F. These images show $\approx 2-3 \mu \mathrm{m}$ thick transverse sections within the intact tendon. They 
display a fibrillar pattern characteristic for the collagen macromolecular organization, in agreement with previous reports (Stoller et al., 2003; Williams et al., 2005; Erikson et al., 2007). They do not directly show the fibers within the tendon fascicles, but correspond to interference patterns. The SHG signal indeed results from the coherent summation of the SHG response of all the fibrils within the focal volume and is more or less intense depending on the relative position and orientation of these fibrils (LaComb et al., 2008; Rivard, et al., 2011; Strupler and Schanne-Klein, 2010).

In most of our experiments, the tendon fascicle exhibited small but well defined crimps at $0 \%$ strain (Fig. 3A). At the ultimate strain of a cycle, the fascicle was always straight, with no visible crimped pattern in intact tendons (Fig. 3B and D). In broken tendons, we observed a stretched region in the center and a crimped region near the tendon edge in the same image. During stretching, the crimps disappear at a given strain, and then reappear at higher strain during relaxation, indicating an inelastic behavior.

In order to quantify the crimp pattern along a loading path, we measured the crimp period in the SHG images. Fig. 3G shows the variation of the crimp period at 0\% strain (along the dotted line for 0\% in Fig. $3 F)$. The period decreases with the cycle number, which means that the crimps are increasingly marked in SHG images (see Fig 3A, C and E) after every cycle. Note that complementary measurements of the crimp amplitude would require 3D reconstruction of the tendon fascicle by recording SHG image stacks instead of only one transverse section. This could result in confused data because of relaxation during the longer pauses which would be needed for SHG imaging.

Fig. $3 \mathrm{H}$ displays the crimp period at $2 \%$ strain versus the cycles with ultimate strain above $4 \%$ (see the dotted line $2 \%$ in fig. $3 F$ ). There are two points per cycle, the lowest one being always in the relaxation part. For cycles of a given series (same ultimate strain - same color in Fig. 3), we observe that the period increases between a relaxation and the next stretch.

We note that the crimp period, shown in Fig 3H, evolves similarly to the lowest measurable strain, shown in Fig $2 \mathrm{E}$ for the same tendon fascicle. Both of them indicate that there is an irreversible deformation of the tendon fascicle during preconditioning.

\section{Discussion}

In this study, we successfully implemented continuous in situ SHG imaging of a tendon subjected to a mechanical assay. SHG microscopy advantageously provided optically sectioned images of the micrometrescale fibrillar organization of the tendon. Our SHG images showed a better contrast than polarized-light microscopy images that integrate the tendon structure over its full thickness. Moreover, the high specificity of SHG microscopy for fibrillar collagen resulted in high-contrast images compared to other 3D techniques like confocal microscopy or optical coherence tomography.

SHG images and mechanical measurements show that preconditioning is associated with a change in the stress-free configuration. It is stronger during the first stretch for a given ultimate strain, although many more cycles are required to reach the limit curve (Abrahams, 1967). Preconditioning is associated both with a shift of the toe-region of the stress-strain curve to higher strains (Fig 2F), and a decrease in the crimp period (Fig 3G and $\mathrm{H}$ ). We attribute this behavior to a sliding of the collagen fibrils with respect to each other, extending the relaxed length of the tendon fascicle. Since SHG observations are sensitive to the fibril scale, our results indicate that sliding occurs at a similar or smaller scale, but they cannot distinguish between fibrillar and intra-fibrillar sliding.

However, the preconditioning is not irreversible. During an extended pause at the initial length of the tendon fascicle, we observed a decrease of the resting length (see Fig 2F). This recovery from previous preconditioning is usually attributed to a reconfiguration of the bonds between fibrils or lower-scale structures (Vesentini et al., 2005; Ciarletta and Ben Amar, 2009). On a shorter time scale, we observe that the crimp period at $2 \%$ strain increases significantly between a relaxation and the next stretching of the tendon fascicle. This was not observed at $0 \%$ strain, which indicates that relaxation involves different time 
scales, and therefore different length scales. We cannot access faster relaxation times than a few tenths of a second, which is the time needed to adjust imaging parameters (Gupta et al., 2010).

All these considerations indicate that preconditioning is mostly due to sliding of microstructures inside the fascicle. SHG imaging shows that a significant part of the sliding occurs between fibrils; previous works showed that sliding occurs between and inside the fibrils (Puxkandl et al., 2002; Screen et al., 2004; Cheng and Screen, 2007). We also observed that the preconditioned state is temporary and can recover, at least partially, on a longer time scale (Hansen et al., 2002).

\section{Conclusion}

We developed a new multi-scale experiment to monitor simultaneously the fibrillar collagen architecture at microscopic scale and the strain-stress relationship at macroscopic scale in biological tissues. To that end, we combined a multiphoton microscope and a mechanical device, and we performed simultaneous SHG imaging and controlled mechanical loading of rat-tail tendon. This biomechanical setup has never been reported yet to the best of our knowledge. Our study provides a proof of concept that this device enables analysis of the connection between macroscopic response and microscopic structure of tissues. We showed that mechanical preconditioning mostly arises from sliding and is reversible on long time-scales. This supports the notion that the mechanical behavior of a tendon fascicle relies on its microstructure remodeling. Our experimental setup can be readily generalized to other mechanical assays and to any other bidimensional tissue, such as skin or cornea. SHG imaging can also be combined with 2PEF imaging to visualize either elastin fibers or labeled cells. Our method should then permit continuous analysis of 3D remodeling of connective tissues in mechanical assays and bring new valuable information to biomechanics of microstructured tissues.

\section{Conflict of interest statement}

All authors have no conflict of interest and nothing to disclose.

\section{Acknowledgments}

We thank Daniel Caldemaison and Viet Tran for their help in the design of the experimental setup.

\section{References}

Abrahams, M., 1967. Mechanical Behaviour of Tendon In Vitro - A Preliminary Report. Med. \& Biol. Engng 5, 433443.

Campagnola, P. J., Millard, A. C., Terasaki, M., Hoppe, P. E., Malone, C. J., Mohler, W. A., 2002. Three-dimensional high-resolution second-harmonic generation imaging of endogenous structural proteins in biological tissues. Biophys. J. 82, 493-508.

Cheng, V. W. T., Screen, H. R. C., 2007. The micro-structural strain response of tendon. J. Mater Sci. 42, 8957-8965.

Ciarletta, P., Ben Amar, M., 2009. A finite dissipative theory of temporary interfibrillar bridges in the extracellular matrix of ligaments and tendons. J. R. Soc. Interface 6, 909-924.

Cox, G., Kable, E., Jones, A., Fraser, I. K., Manconi, F., Gorrell, M. D., 2003. 3-dimensional imaging of collagen using second harmonic generation. J. Struct. Biol. 141, 53-62.

Deniset-Besseau, A., Duboisset, J., Benichou, E., Hache, F., Brevet, P. F., Schanne-Klein, M.-C., 2009. Measurement of the Second-Order Hyperpolarizability of the Collagen Triple Helix and Determination of Its Physical Origin. J. Phys. Chem. B 113, 13437-13445. 
Erikson, A., Ortegren, J., Hompland, T., Davies, C. D., Lindgren, M., 2007. Quantification of the second-order nonlinear susceptibility of collagen I using a laser scanning microscope. J. of Biomed. Optics 12, 044002.

Franchi, M., Fini, M., Quaranta, M., Pasquale, V. D., Raspanti, M., Giavaresi, G., Ottani, V., Ruggeri, A., 2007. Crimp morphology in relaxed and stretched rat Achilles tendon. Journal of Anatomy , 210, 1-7.

Fratzl, P., 2003. Cellulose and collagen: from fibres to tissues. Curr. Opin. Colloid Interface Sci. 8, 32-39.

Fung, D. T., Sereysky, J. B., Basta-Pljakic, J., Laudier, D. M., Huq, R., Jepsen, K. J., Schaffler, M. B., Flatow, E. L., 2010. Second Harmonic Generation Imaging and Fourier Transform Spectral Analysis Reveal Damage in Fatigue-Loaded Tendons. Annals of Biomedical Engineering, 38, 1741-1751.

Freund, I., Deutsch, M., Sprecher, A., 1986. Connective-tissue Polarity - Optical 2nd-harmonic Microscopy, Crossedbeam Summation, and Small-angle Scattering In Rat-tail Tendon. Biophys. J. 50, 693-712.

Fung, D. T., Wang, V. M., Laudier, D. M., Shine, J. H., Basta-Pljakic, J., Jepsen, K. J., Schaffler, M. B., Flatow, E. L., 2009. Subrupture Tendon Fatigue Damage. J. Orthop. Res., 27, 264-273.

Gupta, H. S., Seto, J., Krauss, S., Boesecke, P., Screen, H. R. C., 2010. In situ multi-level analysis of viscoelastic deformation mechanisms in tendon collagen. J. Struct. Biol. 169, 183-191.

Gusachenko, I., Latour, G., Schanne-Klein, M.-C., 2010. Polarization-resolved Second Harmonic microscopy in anisotropic thick tissues. Opt. Express 18, 19339-19352.

Hansen, K. A., Weiss, J. A., Barton, J. K., 2002. Recruitment of tendon crimp with applied tensile strain. J. Biomech. Eng. 124, $72-77$.

Kastelic, J., Galeski, A., Baer, E., 1978. Multicomposite Structure of Tendon. Connect. Tissue Res. 6, 11-23.

Ker, R. F., 2007. Mechanics of tendon, from an engineering perspective. Int. J. Fatigue 29, 1001-1009.

LaComb, R., Nadiarnykh, O., Townsend, S. S., Campagnola, P. J., 2008. Phase matching considerations in second harmonic generation from tissues: Effects on emission directionality, conversion efficiency and observed morphology. Opt. Commun. 281, 1823-1832.

Legare, F., Pfeffer, C., Olsen, B. R., 2007. The role of backscattering in SHG tissue imaging. Biophys. J. 93, 13121320.

Legerlotz, K., Riley, G. P., Screen, H. R. C., 2010. Specimens dimensions influence the measurement of material properties in tendon fascicles. J. Biomech. 43, 2274-2280.

Pena, A.-M., Boulesteix, T., Dartigalongue, T., Schanne-Klein, M.-C., 2005. Chiroptical effects in the second harmonic signal of collagens I and IV. J. Am. Chem. Soc. 127, 10314-10322.

Puxkandl, R., Zizak, I., Paris, O., Keckes, J., Tesch, W., Bernstorff, S., Purslow, P., Fratzl, P., 2002. Viscoelastic properties of collagen: synchrotron radiation investigations and structural model. Philos Trans. R. Soc. Lond. B Biol. Sci. 357, 191-197.

Redaelli, A., Vesentini, S., Soncini, M., Vena, P., Mantero, S., Montevecchi, F. M., 2003. Possible role of decorin glycosaminoglycans in fibril to fibril force transfer in relative mature tendons - a computational study from molecular to microstructural level. J. Biomech. 36, 1555-1569. 
Rigby, B. J., Hirai, N., Spikes, J. D., Eyring, H., 1959. The Mechanical Properties of Rat Tail Tendon. J. Gen. Physiol. 43, 265-283.

Rivard, A., Laliberté, M., Bertrand-Grenier, A., Harnagea, C., Pfeffer, C. P., Vallières, M., St-Pierre, Y., Pignolet, A. ; Khakani, M. A. E., Légaré, F., 2011. The structural origin of second haronic generation in fascia. Biomed. Opt. Express 2, 26.

Screen, H. R. C., Bader, D. L., Lee, D. A., Shelton, J. C., 2004. Local strain measurement within tendon. Strain 40, 157-163.

Screen, H. R. C., Shelton, J. C., Chhaya, V. H., Kayser, M. V., Bader, D. L., Lee, D. A., 2005. The influence of noncollagenous matrix components on the micromechanical environment of tendon fascicles. Ann. Biomed. Eng. 33, 1090-1099.

Sionkowska, A., Wess, T., 2004. Mechanical properties of UV irradiated rat tail tendon (RTT) collagen. Int. J. Biol. Macromol. 34, 9-12.

Stoller, P., Celliers, P. M., Reiser, K. M., Rubenchik, A. M., 2003. Quantitative second-harmonic generation microscopy in collagen. Appl. Opt. 42, 5209-5219.

Stoller, P., Reiser, K. M., Celliers, P. M., Rubenchik, A. M., 2002. Polarization-modulated second harmonic generation in collagen. Biophys. J. 82, 3330-3342.

Strupler, M., Pena, A.-M., Hernest, M., Tharaux, P. L., Martin, J.-L., Beaurepaire, E., Schanne-Klein, M.-C., 2007. Second harmonic imaging and scoring of collagen in fibrotic tissues. Opt. Express 15, 4054-4065.

Strupler, M., Schanne-Klein, M.-C., 2010. Simulating second harmonic generation from tendon: Do we see fibrils? Biomedical Optics, OSA Technical Digest, paper BTuD83.

Vesentini, S., Redaelli, A., Montevecchi, F. M., 2005. Estimation of the binding force of the collagen moleculedecorin core protein complex in collagen fibril. J. of Biomech. 38, 433-443.

Viidik, A., Ekholm, R., 1968. Light and electron microscopic studies of collagen fibers under strain. Anatomy and Embryology, 127, 154-164.

Williams, R. M., Zipfel, W. R., Webb, W. W., 2005. Interpreting second-harmonic generation images of collagen I fibrils. Biophys. J. 88, 1377-1386.

Zipfel, W. R., Williams, R. M., Christie, R., Nikitin, A. Y., Hyman, B. T., Webb, W. W., 2003. Live tissue intrinsic emission microscopy using multiphoton-excited native fluorescence and second harmonic generation. Proc. Natl. Acad. Sci. USA 100, 7075-7080.

Zoumi, A., Yeh, A., Tromberg, B. J., 2002. Imaging cells and extracellular matrix in vivo by using second-harmonic generation and two-photon excited fluorescence. Proc. Natl. Acad. Sci. USA 99, 11014-11019. 


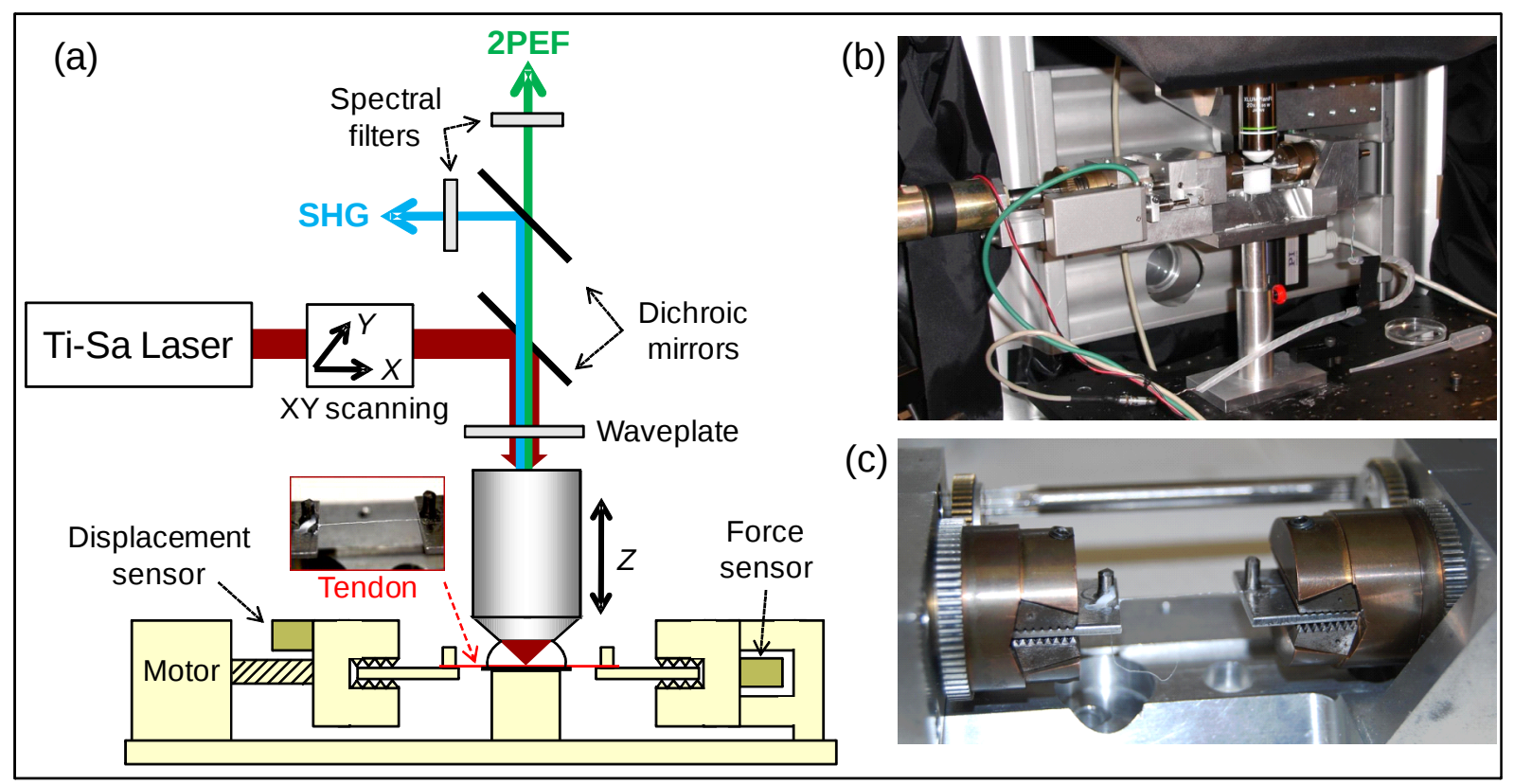

Figure 1 

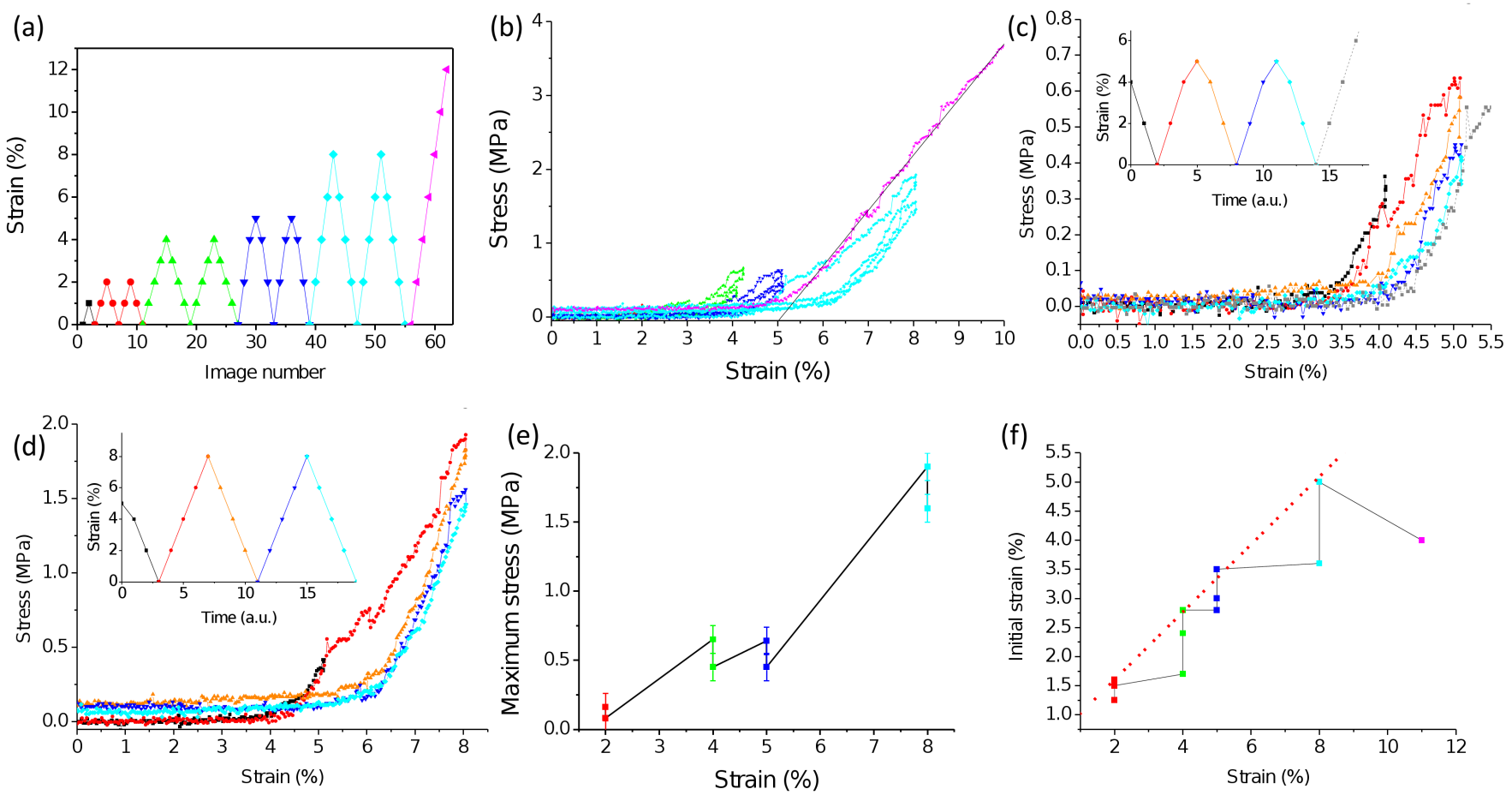

Figure 2 

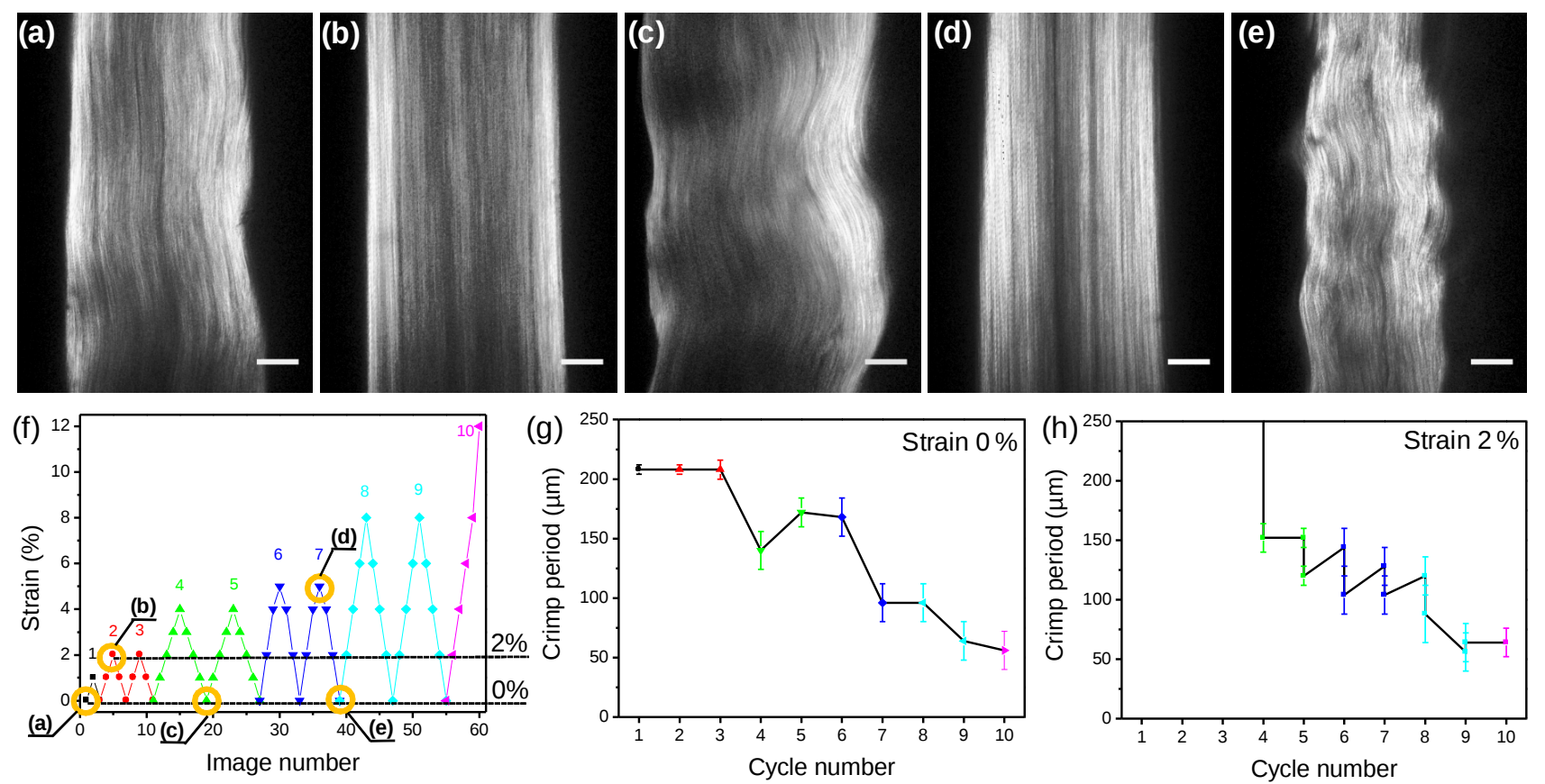

Figure 3 
Figure 1: Experimental setup. (a) Scheme of the laser scanning microscope and the traction device. (b) Picture of the traction device under the objective, showing the tendon immersed in water on a glass coverslip and supported by a teflon base. (c) Zoomed view of the tendon fascicle mount and the grips.

Figure 2: Mechanical behavior of a tendon fascicle. (a) Loading path versus the image number; each color is associated with one ultimate strain. (b) Stress-strain curves for the whole loading path. (c)-(d) Stressstrain curves for a series of cycles at $5 \%$ and $8 \%$ ultimate strain, with color codes as indicated in the insert. (e) Maximum stress per cycle versus the ultimate strain. (f) Initial measurable strain versus the ultimate strain.

Figure 3: SHG imaging of the tendon fascicle microstructure. (a)-(e) SHG images at the time points underlined in orange in (f); scale bars: $50 \mu \mathrm{m}$. (f) Loading path. (g) Evolution of the crimp spatial period at 0 $\%$ strain during the cycles. (h) Same at $2 \%$ strain. These imaging data were recorded simultaneously with the mechanical data displayed in Figure 2, on the same tendon fascicle. 